\title{
Neuroanesthesia Practice during COVID-19: A Single-Center Experience
}

\author{
Rajashree U. Gandhe ${ }^{1}$ \\ ${ }^{1}$ Division of Anaesthesiology, Department of Neuroanesthesiology, \\ Kokilaben Dhirubhai Ambani Hospital and Medical Research \\ Institute, Mumbai, Maharashtra, India
}

\begin{abstract}
Address for correspondence Rajashree U. Gandhe, MD, Division of Anaesthesiology, Department of Neuroanesthesiology, Kokilaben Dhirubhai Ambani Hospital and Medical Research Institute, Mumbai 400053, Maharashtra, India (e-mail: rajashreeug@gmail.com).
\end{abstract}

J Neuroanaesthesiol Crit Care:2020;7:166-169

\begin{abstract}
Keywords

- aerosol-generating procedures

- anesthesia

- COVID-19

- neurointervention

- neurosurgery

The coronavirus disease 2019 (COVID-19) pandemic is a challenge for all health care providers (HCPs). Anesthesiologists are vulnerable to acquiring the disease during aerosol-generating procedures in operating theater and intensive care units. High index of suspicion, detailed history including travel history, strict hand hygiene, use of face masks, and appropriate personal protective equipment are some ways to minimize the risk of exposure to disease. Neurologic manifestations of COVID-19, modification of anesthesia regimen based on the procedure performed, and HCP safety are some implications relevant to a neuroanesthesiologist. National and international guidelines, recommendations, and position statements help in risk stratification, prioritization, and scheduling of neurosurgery and neurointervention procedures. Institutional protocols can be formulated based on the guidelines wherein each HCP has a definite role in this ever-changing scenario. Mental and physical well-being of HCPs is an integral part of successful management of patients. We present our experience in managing 143 patients during the lockdown period in India.
\end{abstract}

\section{Introduction}

Novel coronavirus, severe acute respiratory syndrome coronavirus 2 (SARS-CoV-2; coronavirus disease 2019 [COVID-19]) is a challenge for all health care providers (HCPs) today. It was declared a global pandemic by the World Health Organization on March 11, 2020. ${ }^{1}$ Anesthesiologists are susceptible to contracting the disease during the aerosol-generating procedures in operation theater (OT) and intensive care units (ICUs). High index of suspicion, detailed travel history, strict hand hygiene, use of face masks and, appropriate personal protective equipment (PPE) are some ways to minimize the risk of exposure to the disease. National and international guidelines, recommendations, and position statements help in the risk stratification, prioritization, and scheduling of neurosurgery and neurointervention procedures. ${ }^{2-7}$

Our hospital is a tertiary care center located in suburban Mumbai.Wepresentour experience of anesthetic management of 143 patients ( 76 neurosurgical and 67 neurointerventional) who underwent emergency and urgent procedures between March 25 and June 25, 2020 ( - Table 1 and $\boldsymbol{~ - ~ F i g . ~ 1 ) . ~ E m e r g e n c y ~}$
DoI https://doi.org/ $10.1055 / \mathrm{s}-0040-1721164$ ISSN 2348-0548. (c) 2020. Indian Society of Neuroanaesthesiology and Critical Care. This is an open access article published by Thieme under the terms of the Creative Commons Attribution-NonDerivative-NonCommercialLicense, permitting copying and reproduction so long as the original work is given appropriate credit. Contents may not be used for commercial purposes, or adapted, remixed, transformed or built upon. (https://creativecommons.org/licenses/by-nc-nd/4.0/) 
Table 1 List of neurosurgery and neurointervention procedures

\begin{tabular}{|c|c|}
\hline \multicolumn{2}{|l|}{ Neurosurgery emergency procedures } \\
\hline $\begin{array}{l}\text { Decompressive craniotomy for hematoma } \\
\text { evacuation }\end{array}$ & 7 \\
\hline External ventricular drain & 4 \\
\hline Intracranial pressure monitoring bolt & 1 \\
\hline \multicolumn{2}{|l|}{ Neurointervention emergency procedures } \\
\hline Stroke & 15 \\
\hline Total emergency procedures & 27 \\
\hline \multicolumn{2}{|l|}{ Neurosurgery urgent procedures } \\
\hline Intracranial tumor excision & 18 \\
\hline Awake craniotomy & 3 \\
\hline Chronic subdural hematoma & 10 \\
\hline Navigation/endoscopic biopsy & 4 \\
\hline Endoscopic third ventriculostomy & 1 \\
\hline Cranioplasty & 5 \\
\hline Ventriculoperitoneal shunt & 1 \\
\hline Ommaya reservoir insertion & 1 \\
\hline Spine decompression \pm fusion & 6 \\
\hline Spine tumors & 3 \\
\hline Anterior cervical discectomy and fusion & 3 \\
\hline Tracheostomy & 8 \\
\hline Foramen magnum decompression & 1 \\
\hline \multicolumn{2}{|l|}{ Neurointervention urgent procedures } \\
\hline Angiography & 20 \\
\hline Aneurysm coiling & 9 \\
\hline Arteriovenous fistula/malformation embolization & 1 \\
\hline Carotid stenting & 14 \\
\hline Middle cerebral artery Stenting & 1 \\
\hline Vertebral artery stenting & 7 \\
\hline Total urgent procedures & 116 \\
\hline Grand total (emergency + urgent) & 143 \\
\hline
\end{tabular}

procedures were life-threatening, whereas urgent procedures were not immediately life-threatening but could lead to neurologic damage if not treated.

\section{Case Details}

\section{Preoperative Visit}

All patients underwent a preoperative real-time quantitative polymerase chain reaction (RT-qPCR) analysis of their nasopharyngeal swab, and results were available within 8 hours. A negative report was considered sufficient to proceed for any urgent procedure. The reports were valid for 7 days from sample collection. Guidelines issued by local and national authorities were strictly followed. ${ }^{8}$ Patients were mandated to wear surgical or N95 mask, and PPE was used by the anesthesia team during checkup. We garnered a detailed patient history and list of their associated comorbidity. Noncontact temperature was checked along with resting oxygen saturation $\left(\mathrm{SPO}_{2}\right)$. Basic airway assessment included mouth opening, neck movements, and thyromental distance, which were performed keeping a distance of $2 \mathrm{~m}$ from the patient.

\section{Management of Emergency Procedures}

A total of 27 patients underwent life-saving emergency procedures during this period. These included 12 neurosurgical and 15 neurointervention procedures. Rapid antigen testing and high-resolution computed tomography (HRCT) of the chest were performed in all patients. No patients had HRCT findings suggestive of COVID-19. Care was taken to minimize contamination while transporting these patients from various locations over a short time, and all HCPs were in full PPE for these cases.

\section{Neurosurgical Emergencies}

General anesthesia (GA) with endotracheal intubation was required for 12 neurosurgical emergencies. These included two COVID-19 positive patients requiring subdural hematoma evacuation. Rapid sequence intubation using videolaryngoscope, aerosol box, and a positive air-purified respirator was performed within a negative-pressure OT. Bag-mask ventilation was avoided due to the inherent risk of aerosol generation, and heat-moisture exchange filters were placed to minimize contamination. Balanced anesthesia was provided using inhalational and intravenous anesthetics. Intraoperative hemodynamic and ventilatory parameters remained stable in all patients. All patients were shifted to ICU for elective ventilation using transport ventilators. One COVID-19 positive patient died due to COVID-associated respiratory complications.

\section{Neurointervention Emergencies}

Fifteen patients required emergency endovascular treatment for anterior circulation acute ischemic stroke. These included three patients who tested positive for COVID-19. Decision regarding the type of anesthesia was taken based on the Glasgow Coma Scale (GCS), airway status on admission, and consensus statements. ${ }^{7,9,10}$ All patients were managed with intravenous sedation as their GCS was $>10$ and were able to protect their airway. Patients were spontaneously breathing throughout the procedure with oxygen administered at $<6 \mathrm{~L} /$ minute through face mask placed over the surgical mask. No patient required an urgent conversion to GA or airway intervention. All HCPs wore lead gowns prior to the "donning" of a PPE. Persistent reocclusion of the middle cerebral artery was observed in one COVID-19 positive patient, which was attributed to hypercoagulability of the blood, resulting in the worsening of neurologic condition and subsequent death. The complete recanalization of the affected blood vessels was achieved in the remaining 14 patients.

\section{Management of Urgent Procedures}

Urgent procedures were performed in 64 neurosurgical and 52 neurointervention patients. All patients were documented to be negative for COVID-19. Seventy two patients required GA, while the remaining patients were managed under local anesthesia with intravenous sedation using midazolam, propofol, and dexmedetomidine infusion. Rapid sequence intubation was performed for patients requiring GA in OT or interventional suite. Balanced anesthesia was administered using inhalational and intravenous anesthetics with extubation 


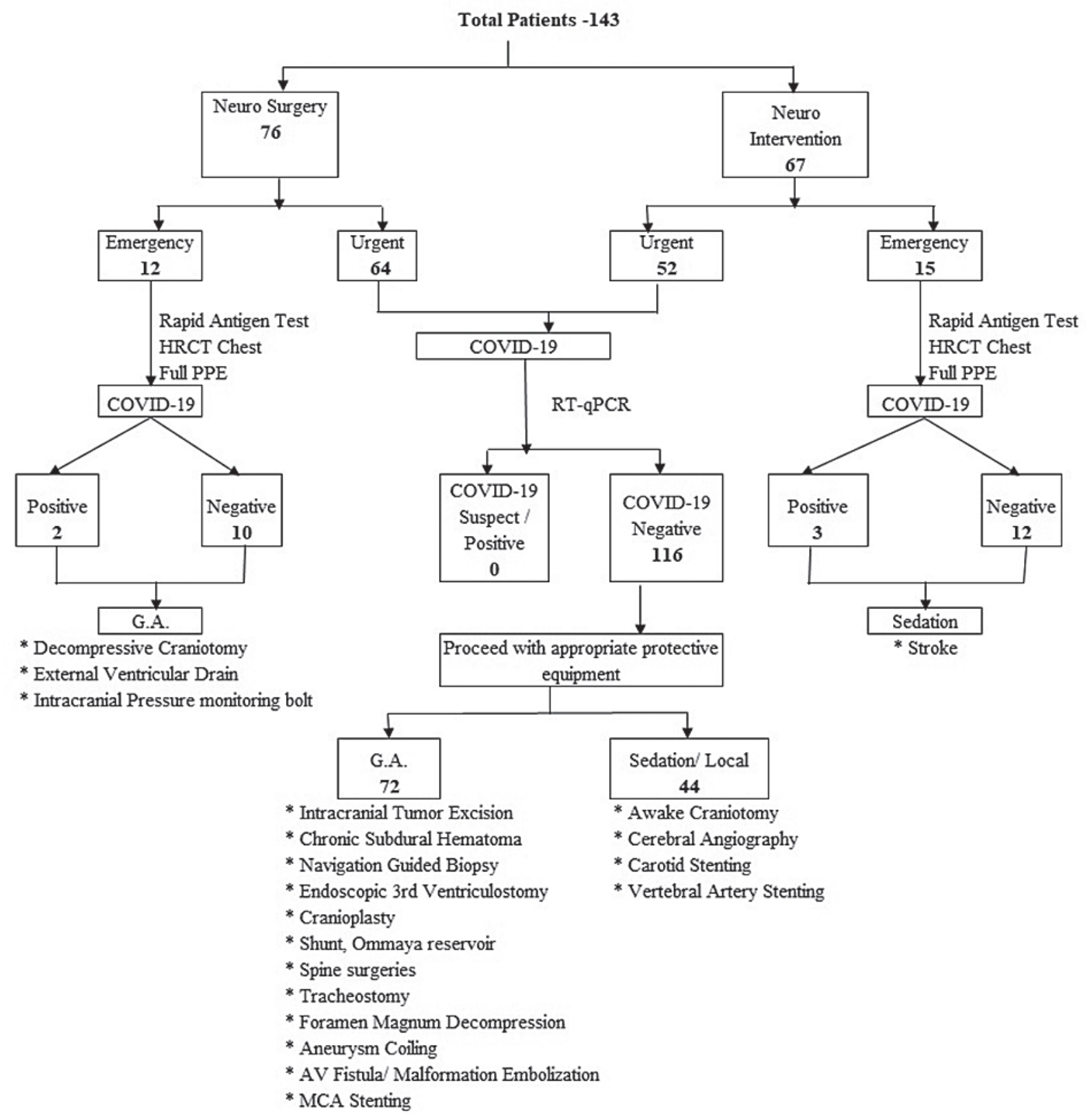

Fig. 1 Flowchart depicting procedures. AV, arteriovenous; COVID-19, coronavirus disease 2019; GA, general anesthesia; HRCT, high-resolution computed tomography; MCA, middle cerebral artery; PPE, personal protective equipment; RT-qPCR, real-time quantitative polymerase chain reaction.

after complete recovery from anesthesia. Protective measures, including aerosol box, face shields, goggles, and transparent drapes, were used during intubation and extubation. Patients managed with intravenous sedation included three patients undergoing awake craniotomy for tumor excision, carotid artery stenting, vertebral artery stenting, and cerebral angiography. No patient managed with intravenous sedation required emergency conversion to GA or airway intervention.

\section{Discussion}

Neuroanesthesia practice at our institute evolved as recommendations for the management of patients during COVID-19 pandemic were published. Institutional protocols were made regarding patient screening, triaging, and procedure prioritization in the setting of limited manpower resources. Dedicated COVID19 ICUs and holding areas were created for the management of COVID positive patients and for those awaiting COVID test results, respectively. HCP teams were created for managing patients during the pandemic with comprehensive training regarding safety given to each member. Physical distancing, strict hand hygiene, use of face masks, and "donning and doffing" of PPE were strictly followed by HCPs to minimize exposure. Emergency procedures were performed in a dedicated negative-pressure OT or interventional suite with all HCPs in full PPE. Negative-pressure OT was created with help of the engineering and maintenance departments of our institute. Care was taken to ensure adequate hydration of all HCPs prior to emergency procedures to prevent dehydration and fatigue in negative-pressure surroundings while managing these cases. Ventilatory circuit disconnections were 
avoided while positioning the patients prone for intracranial tumor excision and spine surgeries. Adherence to national and international guidelines and strict following of protocols helped in preventing disease spread to attending HCPs.

\section{Limitation}

Transnasal endoscopic surgeries were not performed during this period due to reports of increased risk of HCP exposure to COVID-19 virus during these procedures.

\section{Conclusion}

The ongoing COVID-19 pandemic has resulted in significant morbidities and mortalities worldwide with the number of infected people continuously increasing. Neurosurgery and neurointervention procedures need early intervention, and it is essential that the practice of neuroanesthesia evolves with the changing scenario and procedure-specific considerations. Guidelines issued by statutory bodies have helped formulate strategies for the management of these patients. The safety of all HCPs is of paramount importance and should be protected by strictly following all protocols and guidelines.

\section{Conflict of Interest}

None declared.

\section{References}

1 World Health Organization. Coronavirus disease (COVID-19) pandemic. Available at: https://www.who.int/emergencies/ diseases/novel-coronavirus-2019. Accessed July 7, 2020

2 Nedunchezhian AS, Ajayan N, P APH, Prathapadas U, Sethuraman M, Koshy T. Finding the calm in the chaos: an institutional protocol for anesthetic management of a patient for neurosurgery during COVID19 pandemic. J Neurosci Rural Pract 2020;11(3):369-374
3 MOHFW. Ministry of Health and Family Welfare Directorate General of Health Services. [Emergency Medical Relief]. Available at: https://www.mohfw.gov.in/pdf/pdated additional guidelinesonrationaluseofPersonalProtectiveEquipmentsettingapproachforHealthfunctionariesworkinginnonCOVID19 areas.pdf. Accessed July 7, 2020

4 Jangra K, Manohar N, Bidkar PU, et al. Indian Society of Neuroanaesthesiology and Critical Care (ISNACC) position statement and advisory for the practice of Neuroanesthesia during COVID-19 pandemic. J Neuroanaesthesiol Crit Care 2020. Doi: $10.1055 / \mathrm{s}-0040-1714186$

5 Malhotra N, Joshi M, Datta R, Bajwa SJ, Mehdiratta L. Indian Society of Anaesthesiologists (ISA National) Advisory and Position Statement regarding COVID-19. Indian J Anaesth 2020;64(4):259-263

6 Flexman AM, Abcejo AS, Avitsian R, et al. Neuroanesthesia practice during the COVID-19 pandemic: recommendations from Society for Neuroscience in Anesthesiology and Critical Care (SNACC) J Neurosurg Anesthesiol 2020;32(3):202-209

7 Sharma D, Rasmussen M, Han R, et al. Anaesthetic management of endovascular treatment of acute ischemic stroke during COVID-19 pandemic: Consensus statement from Society for Neuroscience in Anaesthesiology and Critical Care (SNACC) J Neurosurg Anesthesiol 2020;32(3):193-201

8 Indian Council of Medical Research. Advisory on use of Rapid Antigen Detection Test for COVID-19. Available at: https:// www.icmr.gov.in/pdf/covid/strategy/Advisory_for_rapid_ antigen_test14062020.pdf. Accessed July 8, 2020

9 Avula A, Nalleballe K, Narula N, et al. COVID-19 presenting as stroke. Brain Behav Immun 2020;87:115-119

10 Talke PO, Sharma D, Heyer EJ, Bergese SD, Blackham KA, Stevens RD. Society for Neuroscience in Anaesthesiology and Critical Care expert consensus statement: anaesthetic management of endovascular treatment for acute ischemic stroke. J Neurosurg Anesthesiol 2014;26:95-108 